\title{
Taquicardia Ventricular Sustentada como Apresentação Isolada de Cardiomiopatia Amiloidótica por Transtirretina - Val50Met
}

\author{
Sustained Ventricular Tachycardia as an Isolated Presentation of Transthyretin Amyloidosis \\ Cardiomyopathy - Val50Met
}

Edileide de Barros Correia, ${ }^{1 \oplus}$ Larissa Ventura Ribeiro Bruscky, ${ }^{1 \oplus}$ Kelin Chen, ${ }^{1 \oplus}$ Priscila Cestari Quagliato, ${ }^{10}$ Yoná Afonso Francisco, ${ }^{1 \oplus}$ Líria Maria Lima da Silva, ${ }^{1}$ Ana Cristina de Souza Murta ${ }^{1 \odot}$

Instituto Dante Pazzanese de Cardiologia, Centro de Amiloidose Cardíaca, ${ }^{1}$ São Paulo, SP - Brasil

\section{Introdução}

A amiloidose é caracterizada por deposição extracelular de proteínas insolúveis (depósito amilóide) em múltiplos órgãos e disfunção orgânica progressiva. Classifica-se pelo tipo de proteína que se deposita. Entre elas, a amiloidose por transtirretina (ATTR), que pode ocorrer por uma variante genética e pelo envelhecimento. ${ }^{1}$ Cerca de 120 variantes genéticas são reconhecidamente a causa da ATTR entre elas, uma das mais comuns é a representada pela Val50Met. A apresentação fenotípica da amiloidose TTR pela mutação Val50Met é majoritariamente neurológica, chamada de polineuropatia amiloidótica familiar (PAF), porém o envolvimento cardíaco está cada vez mais sendo reconhecido. ${ }^{2}$ Casos esporádicos com manifestação estritamente cardiológica, ocasionando hipertrofia da parede ventricular, disfunção diastólica e distúrbios de condução já são descritos na literatura. ${ }^{3}$

Neste artigo, relatamos um caso de ATTR pela mutação Val50Met com a expressão fenotípica de acometimento cardíaco, com taquicardia ventricular sustentada como apresentação isolada.

\section{Relato do Caso}

Brasileiro de 61 anos, hipertenso, filho de pais portugueses, queixando-se de dor torácica seguida de palpitações uma hora antes de chegar ao pronto socorro. Apresentava-se com sudorese intensa, frequência cardíaca elevada (196 batimentos por minuto) e pressão arterial em repouso de 130/80 mmHg. O eletrocardiograma revelou taquicardia ventricular sustentada (Figura 1). Foi prontamente submetido à cardioversão elétrica com sucesso e dois dias após foi implantado um cardiodesfibrilador interno (CDI). Na investigação etiológica, foram realizados vários exames descritos a seguir: marcadores

\section{Palavras-chave}

Amiloidose Familiar; Cardiomiopatia Restritiva; Taquicardia Ventricular.

\section{Correspondência: Edileide de Barros Correia}

Avenida Dante Pazzanese, 500. CEP: 04012-180, Ibirapuera,

São Paulo, SP - Brasil.

E-mail: edileide.barros@dantepazzanese.org.br

Artigo recebido em 06/09/2021, revisado em 24/09/2021, aceito em 24/09/2021.

DOI: https://doi.org/10.36660/abchf.20210015 de necrose miocárdica mostraram-se elevados, em curva ascendente (pico de CK-MB: 31,3 ug/L e troponina-US: $19.400 \mathrm{ng} / \mathrm{ml}$ ); cineangiocoronariografia revelou lesão de 30\% em artéria circunflexa e área luminal de $8,6 \mathrm{~mm}^{2}$ (ultrassom intracoronariano); ecocardiograma mostrou fração de ejeção de $66 \%$, aumento de espessura de paredes (septo $19 \mathrm{~mm}$ e parede posterior $15 \mathrm{~mm}$ ), aumento da espessura do ventrículo direito (VD) e septo interatrial, ventrículo esquerdo (VE) com 52/33 mm, aumento atrial moderado (volume atrial esquerdo indexado de $45 \mathrm{ml} / \mathrm{m}^{2}$ ), disfunção diastólica grave (grau III) e pressão sistólica de artéria pulmonar (PSAP) de $45 \mathrm{mmHg}$. Apesar da fração de ejeção normal, o strain longitudinal global evidenciou comprometimento da função sistólica do VE de -9 (valor de referência: -18), poupando a porção apical.

A ressonância magnética mostrou a maior espessura na parede média inferosseptal do ventrículo esquerdo (22 mm), realce tardio difuso com gadolínio de padrão não isquêmico, circunferencial e edema miocárdico. (Figura 2) A pesquisa de cadeias leves, relação kappa/lambda, imunofixação sérica e urinária foram negativas e a cintilografia com pirofosfato demonstrou intensa captação do pirofosfato marcado com tecnécio simétrica nas paredes do VE e de intensidade leve no VD, com contagem do hemitórax esquerdo sobre o hemitórax contralateral de 2,3 (estudo positivo $>1,5$ ). O teste genético evidenciou uma mutação no gene da transtirretina p.Val50Met.

O paciente evoluiu com perda de peso, síndrome do túnel do carpo bilateral e diminuição discreta de força distal de membros inferiores, sintomas compatíveis com comprometimento do sistema nervoso periférico e do sistema nervoso autônomo. Iniciou tratamento com tafamidis em novembro de 2019. Um ano após, apresentou sintoma de dispneia aos esforços, sendo observada ao exame físico, a presença de hepatomegalia e turgência jugular. Foi medicado com dose baixa de diurético por três dias e foi aumentada a frequência cardíaca do seu cardiodesfibrilador implantável para 80 bpm, com resolução dos sintomas. Na evolução apresentou uma terapia apropriada do CDI por taquicardia ventricular.

Evoluiu assintomático, referindo apenas lentificação dos seus passos ao subir ladeiras íngremes. Repetiu o ecocardiograma um ano e meio após o início do uso do tafamidis que evidenciou uma fração de ejeção de 56\%, espessura septal de $18 \mathrm{~mm}$ e de parede posterior de $16 \mathrm{~mm}$, aumento da espessura de VD e septo interatrial, VE 51/36 $\mathrm{mm}$, volume atrial moderado (volume atrial esquerdo indexado de $42 \mathrm{ml} / \mathrm{m}^{2}$ ), disfunção diastólica grau II, PSAP de $23 \mathrm{mmHg}$, strain longitudinal global de $-7,7$. 


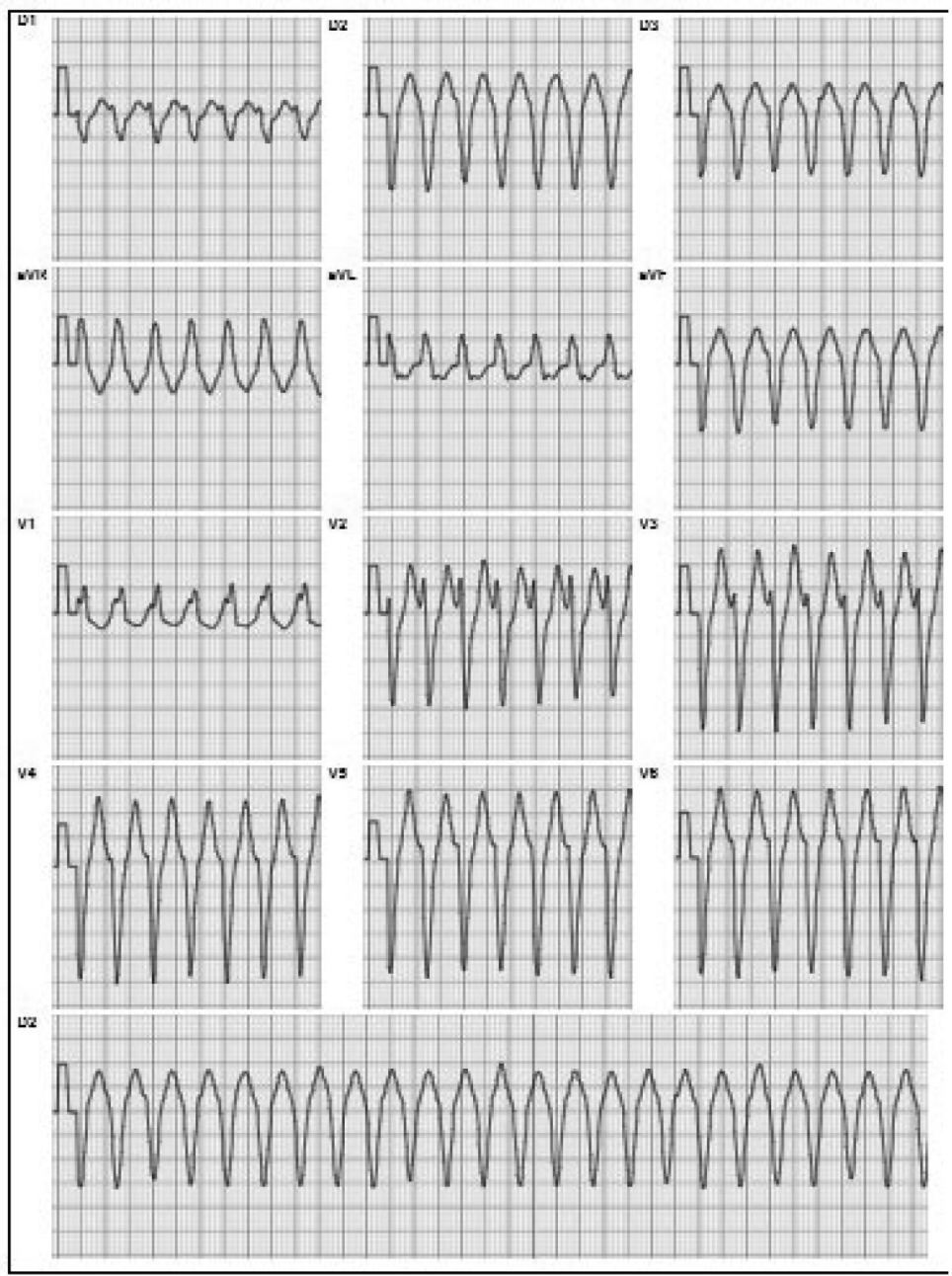

Figura 1 - Eletrocardiograma com taquicardia ventricular sustentada.

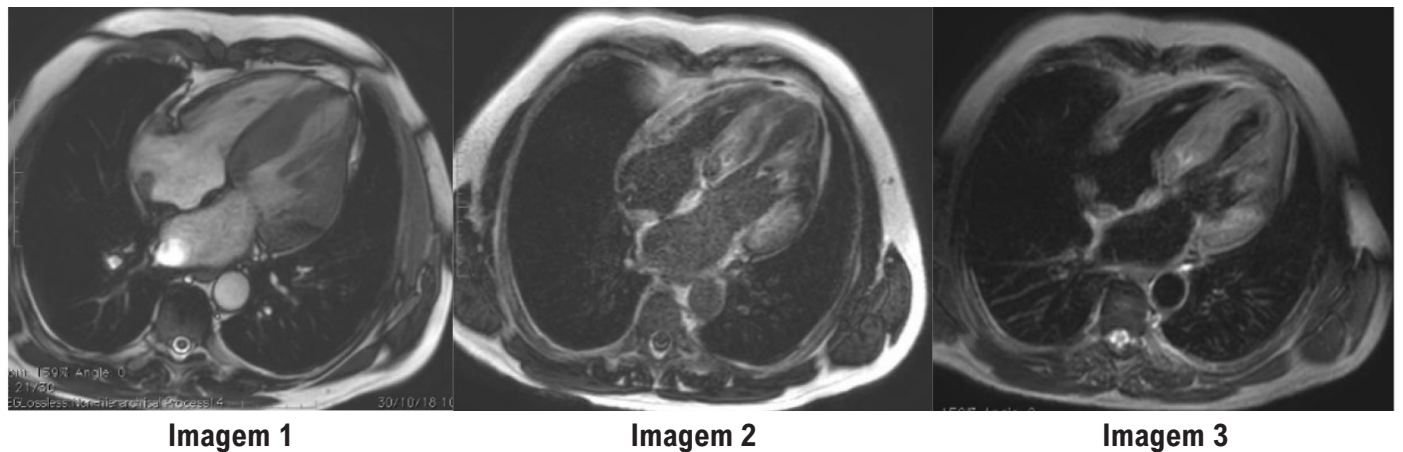

Figura 2 - Ressonância magnética cardiaca evidenciando na imagem 1: cine 4 câmaras; imagem 2: realce tardio 4 câmaras; imagem 3: Tripple BB edema. 


\section{Discussão}

Taquiarritmia ventricular em paciente idoso com elevação em curva dos níveis de troponina é altamente indicativo de quadros de síndrome coronariana aguda. ${ }^{4}$ No presente caso, esta foi a primeira hipótese diagnóstica considerada, no entanto, a cinecoronariografia realizada afastou esta possibilidade. O ecocardiograma ao demonstrar a presença de aumento de espessura importante tanto em septo como em parede lateral, nos desvia do diagnóstico de quadros inflamatórios pela magnitude da hipertrofia e nos leva ao diagnóstico de um grupo de doenças que se apresentam com aumento de espessura de parede, a cardiomiopatia hipertrófica, a mais prevalente após a hipertensiva, a doença de Fabry e a amiloidose cardíaca. ${ }^{5} \mathrm{O}$ aumento de espessura de parede associado aos outros achados encontrados no ecocardiograma como disfunção diastólica grave, apical sparing e redução significativa do strain longitudinal, típicos de amiloidose, nos aponta na direção deste diagnóstico. A ressonância de coração, além de confirmar estes achados, acrescenta mais informações características desta doença, o realce tardio circunferencial e edema miocárdico. O achado de edema miocárdico tem sido relacionado à doença aguda, é mais comum na amiloidose de cadeias leves e está associado à ocorrência de complicações graves. ${ }^{6}$ No presente caso, a ocorrência de taquicardia ventricular pode ser relacionada a este substrato estrutural.

O implante de CDI nos pacientes com amiloidose tem indicações conflitantes nos estudos realizados antes do início da terapêutica específica, modificadora de sobrevida. O caráter progressivo da doença, com perda evolutiva da função ventricular pode justificar a não demonstração do benefício do implante. No entanto, agora com a introdução do tratamento específico, principalmente se o paciente se encontra numa fase mais precoce de evolução da doença, em classe funcional I/II da NYHA é provável que esta recomendação seja modificada em diretrizes, por ser possível a expectativa de sobrevida razoável. ${ }^{1}$

\section{Conclusão}

A Val50Met é uma mutação patogênica caracterizada, principalmente, por ser clinicamente expressa por PAF. Cardiomiopatia, nefropatia e opacificação do humor vítreo podem estar presentes, mas a PAF é a apresentação principal. Quando a cardiomiopatia está presente, geralmente insuficiência cardíaca, mas sem arritmia ventricular, é a apresentação principal. No paciente descrito, a arritmia ventricular era a única manifestação e amiloidose nem mesmo foi considerada no diagnóstico diferencial, até a ressonância magnética. Este caso destaca o risco de morte cardíaca súbita em pacientes com ATTR hereditária. Embora a associação entre ATTR hereditária e morte súbita seja bem reconhecida, os estudos não mostraram nenhum benefício de sobrevida com o implante de desfibriladores implantáveis, talvez devido ao atraso no diagnóstico. A ATTR hereditária como causa da taquicardia ventricular sustentada deve então ser considerada.

\section{Agradecimentos}

Agradecimento especial aos membros do centro de amiloidose e do setor de miocardiopatias do Instituto Dante Pazzanese de Cardiologia por prestar serviço médico à comunidade e contribuir para o desenvolvimento de pesquisa nessa área de doenças negligenciadas da saúde brasileira. Agradecimento à direção do hospital e as equipes médicas setoriais pelo trabalho em conjunto em prol do paciente, colaborando com a realização de exames complementares e análise conjunta do caso clínico. Agradecimento especial aos pacientes que depositam sua confiança na equipe multidisciplinar do hospital para cuidado com sua saúde e possibilitam um aperfeiçoamento contínuo.

\section{Contribuição dos autores}

Concepção e desenho da pesquisa e Revisão crítica do manuscrito quanto ao conteúdo intelectual importante: Correia EB, Bruscky LVR; Obtenção de dados: Correia EB, Bruscky LVR, Silva LML, Murta ACS; Análise e interpretação dos dados: Correia EB, Bruscky LVR, Chen K, Quagliato PC; Redação do manuscrito: Correia EB, Bruscky LVR, Francisco YA, Silva LML.

\section{Potencial Conflito de Interesse}

Declaro não haver conflito de interesses pertinentes.

\section{Fontes de Financiamento}

O presente estudo não teve fontes de financiamento externas.

\section{Vinculação Acadêmica}

Não há vinculação deste estudo a programas de pósgraduação.

\section{Aprovação Ética e consentimento informado}

Este artigo não contém estudos com humanos ou animais realizados por nenhum dos autores. 


\section{Referências}

1. Simões MV, Fernandes F, Marcondes-Braga FG, Scheinberg P, Correia EB, Rohde LEP, et al. Posicionamento sobre Diagnóstico e Tratamento da Amiloidose Cardíaca - 2021. Arq. Bras. Cardiol. 2021;117(3):561-98. doi: 10.36660/abc. 20210718 .

2. Yilmaz A, Bauersachs J, Bengel F, Büchel R, Kindermann I, Klingel K, et al. Diagnosis and Treatment of Cardiac Amyloidosis: Position Statement of the German Cardiac Society (DGK). Clin Res Cardiol. 2021;110(4):479-506. doi: 10.1007/s00392-020-01799-3.

3. Cordeiro CP, Pozzatti RR, Ferreira SG, Freitas MS, Rocha AM, Ribeiro ML, et al. Cardiomyopathy Related to Transthyretin Val30met Mutation in Hereditary Systemic Amyloidosis. Arq Bras Cardiol. 2015;28(2):104-8. doi: 10.5935/2318-8219.20150012.
4. Towbin JA, McKenna WJ, Abrams DJ, Ackerman MJ, Calkins H, Darrieux FCC, et al. 2019 HRS Expert Consensus Statement on Evaluation, Risk Stratification, and Management of Arrhythmogenic Cardiomyopathy. Heart Rhythm. 2019;16(11):301-72. doi: 10.1016/j.hrthm.2019.05.007.

5. Bruscky LVR, Murta ACS, Albrecht FC, Magalhães MJL, Borges Filho R, Francisco YA. Differential Diagnosis among Cardiomyopathies that evolve with ventricular hypertrophy. Rev Soc Cardiol Estado de São Paulo. 2021;31(2):171-80. doi: http://dx.doi.org/10.29381/0103$8559 / 20213102171-80$

6. Fontana M, Corović A, Scully P, Moon JC. Myocardial Amyloidosis: The Exemplar Interstitial Disease. JACC Cardiovasc Imaging. 2019;12 (11):234556. doi: 10.1016/j.jcmg.2019.06.023. 